\title{
Categorías léxicas y clasificación nominal en muinane. Noroeste Amazónico.
}

\author{
Lexical categories and nominal classification in muinane. \\ Northwest Amazon.
}

Consuelo Vengoechea ${ }^{1}$

\begin{abstract}
Resumen
El Predio Putumayo es un resguardo indígena situado en la región amazónica colombiana. Este territorio y sus alrededores están habitados por múltiples grupos indígenas que se comunican en diversas lenguas. Estos grupos indígenas se diferencian e identifican lingüísticamente y mantienen estrechas relaciones interétnicas y matrimoniales entre sí. En los bordes del territorio se encuentran también de manera ocasional algunos asentamientos familiares de gente arawak o tukano. Los vecinos del Resguardo Predio Putumayo hablan lenguas de las familias Tukano oriental, centro oriental, Tukano occidental y Arawak. Contamos con pocos estudios comparativos de las prácticas culturales y lingüísticas del interfluvio Caquetá-Putumayo y aquéllas de la cuenca del Alto río Vaupés. En este artículo se analiza la relación existente entre categorías léxicas y el sistema de clasificación nominal del muinane en perspectiva comparativa procurando así describir el sistema y sus funciones. Finalmente planteamos ampliar estas descripciones tomando en cuenta la relación existente entre la ausencia de una categoría adjetival y la presencia de los sistemas de clasificación nominal para un grupo de lenguas habladas en la región del Noroeste amazónico.

Palabras clave: Categorías léxicas. Clasificación nominal. Muinane. Familias linguísticas. Bora. Tukano. Uitoto.
\end{abstract}

\begin{abstract}
The Predio Putumayo is an indigenous territory located in the Colombian Amazon. Villages of divers indigenous groups speaking different languages are dispersed in this landscape and in their periphery. Those groups identify themselves by using linguistic and other cultural differentiations. They maintain inter-ethnical and closer relationships by common festivals and marriage. Occasionally, some familiar settlements from Tukano and Arawak people are found in the borders of this territory. Neighbours from the Resguardo Predio Putumayo speak languages from eastern, and western Tukano and Arawak families. We have account of few comparative studies of the social and linguistic practices from the CaquetáPutumayo inter-fluvial region and those of the Vaupés region. This article propose to study the relationship between lexical categories and nominal classification system in Muinane language, looking for describe this system and their functions and comparing Muinane with other languages near around. We propose a wider description taking in account the relation between the lack of an adjective class and the appearance of prominent systems of nominal

${ }^{1}$ Profesora Catedrática Asociada, Universidad Nacional de Colombia. Departamento de Antropología. Bogotá, Colombia. Doctora Ciencias del Lenguaje, Universidad de Toulouse II, Le Mirail. Francia. MA Etnolingüística y Antropóloga Universidad de Los Andes, Colombia.
\end{abstract}


classification in a group of languages spoken in the Northwest Amazon.

Keywords: Lexical categories. Nominal classification. Muinane language. Linguistic families. Bora. Tukano. Uitoto.

\section{Introducción}

En este artículo presentamos en $\S 2$ algunas características generales del grupo muinane, su territorio tradicional y su situación social e histórica. En $\S$ 3 hablamos de la pertenencia lingüística de la lengua muinane y describimos sintéticamente sus rasgos tipológicos para continuar en $\S 4$ previa presentación de las características más sobresalientes de la lengua muinane, indagando sobre la relación existente entre las categorías léxicas y el sistema de clasificación nominal de esta lengua de la familia Bora. Se contrastan los resultados en $\S 5$ con los presentados por otros investigadores que se han ocupado de las lenguas de misma familia. En $\S 6$ se comparan los aspectos estudiados entre lenguas de las familias Bora, Uitoto, Tukano oriental y Tucano centro oriental. Finalmente, en $\S 7$ abordamos algunas consideraciones antropológicas y lingüísticas finales, proponiendo ampliar el espectro de nuestras interpretaciones sobre los sistemas de clasificación nominal en la región del Noroeste amazónico

\section{El grupo muinane, territorio y situación actual}

Los muinanes y otros grupos étnicos habitan en el Noroeste Amazónico en diferentes territorios indígenas reconocidos por el gobierno colombiano como son los resguardos Predio Putumayo y Monochoa. Estas extensiones son constitutivas del territorio ancestral de la "gente de centro", en el cual se incluyen los grupos muinane, uitoto, andoque, bora, miraña, ocaina y nonuya. Una buena parte de la población del conglomerado ha migrado en los últimos sesenta años desde el interior de la selva hacia las riberas de los grandes ríos y hacia los centros de población intermedios o mayores. Este proceso migratorio es la respuesta a las acciones económicas foráneas relacionadas con el avance de la globalización y de la economía de mercado, las cuales han influido en la aparición de diversos conflictos sociales que han tenido lugar en la región. La explotación del caucho a principios del siglo XX estuvo acompañada de un etnocidio y esclavización crecientes de las poblaciones indígenas de parte de los comerciantes y caucheros, cuyas consecuencias llevaron al conflicto colombo peruano de los años treinta. Posteriormente otros procesos de extracción de recursos, minerales y de cultivos ilícitos han azotado la región desde la segunda mitad del siglo XX hasta el presente. Un factor adicional que ha motivado la migración es el conflicto armado colombiano de las últimas décadas. Algunos grupos familiares o de jóvenes muinane han migrado hacia los centros urbanos en los últimos veinte años. A pesar de las consecuencias de la historia reciente, 
una parte de la población muinane continúa habitando áreas del territorio y sus alrededores. Estos grupos indígenas continúan ocupando las riberas de los medianos y grandes ríos de la selva húmeda tropical.

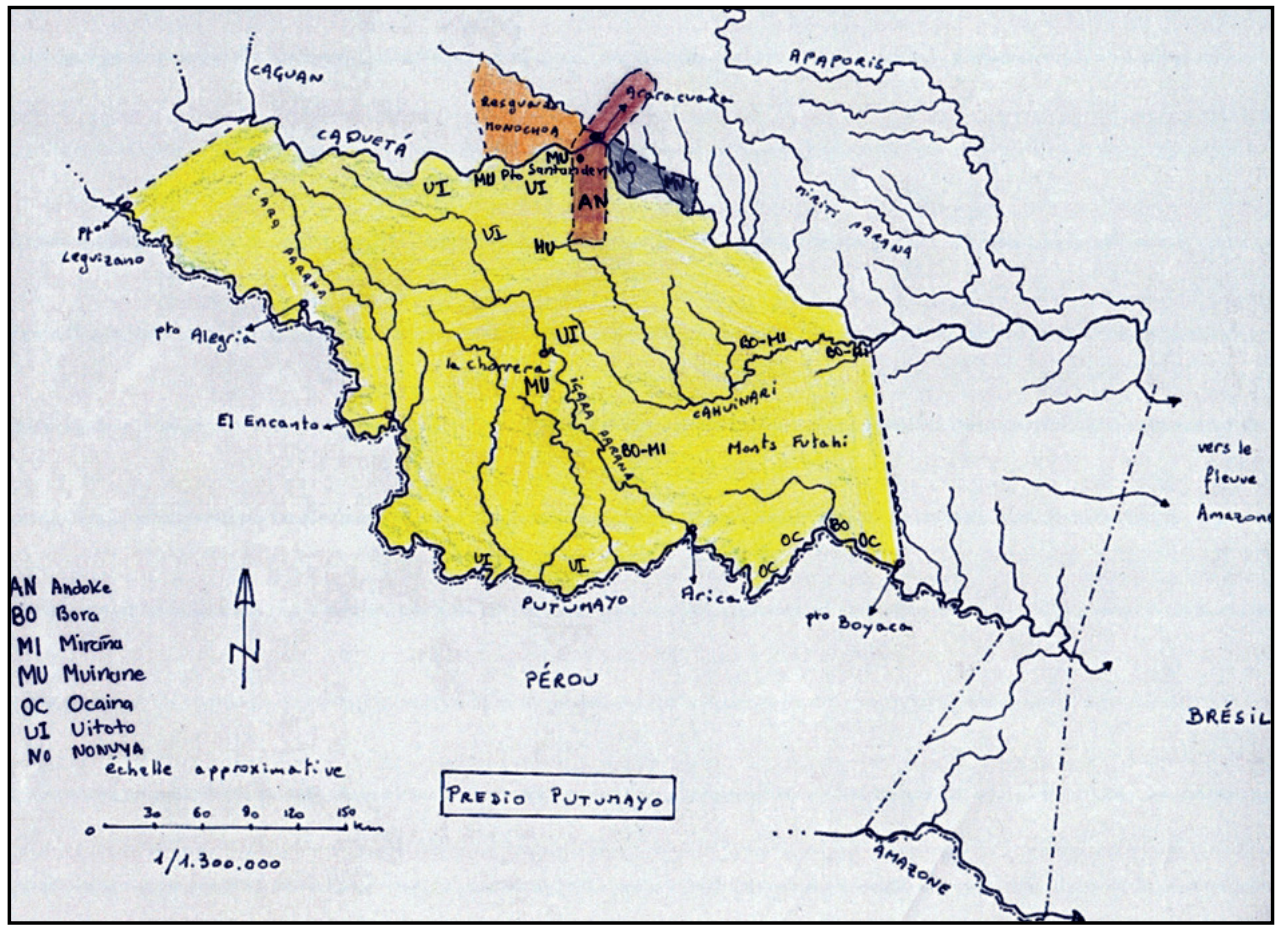

La región Caquetá Putumayo en la Amazonía colombiana. Fuente: Ministerio de Educación Nacional de Colombia. Atlas Indigenista Bogotá: Arco, 1977. Reelaborado y traducido al Francés por el Geógrafo Alain Bunge, 2012. Tomado de Vengoechea (2012:13)

\section{Pertenencia lingüística y rasgos tipológicos}

La lengua muinane ha sido clasificada como perteneciente a la familia lingüística Bora. Landaburu (2000:41) ha propuesto estudiar y comparar, en primer lugar las lenguas de esta familia: el muinane, miraña y el bora propiamente dicho. En segundo lugar, las lenguas Uitoto para proceder así a una reclasificación científica. Los criterios propuestos por Landaburu son gramaticales, léxicos, tipológicos y geográficos. Estas investigaciones se están llevando a cabo por varios autores (Fagua, Petersen de Piñeres, Seifart, Vengoechea, Walton, entre otros), aunque son necesarios mayores esfuerzos comparativos para las diversas lenguas de la región. Para Aschmann (1993), citado en Seifart (2007:412) y para el mismo Seifart, las tres lenguas: muinane, bora y miraña pertenecerían a la familia lingüística Uitoto. Sin embargo, nosotros preferimos la propuesta de Landaburu (2000:40-41) ya que no tenemos estudios comparativos suficientes. 
Los rasgos tipológicos del muinane, en lo que concierne a la fonología, son su prosodia sobresaliente constituida por la tonía, el peso moraico, la armonía palatal, de altura vocálica y glotal. La sílaba en su estructura no posee coda pero su núcleo puede ser liviano o pesado. Varias lenguas de la región amazónica, de familias Bora, Uitoto y Tukano oriental o centro oriental, comparten sistemas prosódicos complejos. Las lenguas bora y miraña de la familia Bora y las lenguas de familia Tukano oriental como el tatuyo y el barasana presentan tonía, peso moraico, así como diferentes tipos de armonía. En cuanto a su morfología, el muinane es una lengua aglutinante y posee también una serie de estructuras polisintéticas. Las unidades léxicas pueden mostrar un buen número de sufijos, siendo los únicos prefijos sobre aquéllas, las marcas de persona. El sistema de clasificación nominal es prominente. Lo registramos con valor tanto léxico, como morfosintáctico y discursivo. Estos sistemas se han documentado para las lenguas Bora (Vengoechea 2001 y 2003), (Seifart 2003, 2005, 2007), Uitoto (Petersen de Piñeres 2007), Tucano (Gomez-Imbert 1982, 2007, 2011) y (Brandrup 2000), Arawak (Aikhenwald 2000), y Peba-yagua (Payne 2007). En la sintaxis, la clasificación nominal juega un papel importante en cuanto aporta en muinane marcas e índices argumentales. La lengua muestra un claro alineamiento nominativo acusativo en las oraciones perifrásticas, es decir, en las oraciones construidas con más de un término o palabra. Sin embargo, en oraciones holofrásticas el tipo de alineamiento sintáctico para la tercera persona es absolutivo ergativo (Vengoechea 2013:150-162). Por oraciones holofrásticas entendemos aquéllas relaciones predicativas en las cuales el núcleo de la predicación y sus argumentos están incluidos en un único término o palabra (cf. ejs., (5) y (6)). Las lenguas de la familia Bora, así como aquéllas mencionadas aquí de la familia Tukano oriental y Tukano centro oriental, son principalmente sufijales y exhiben en su morfosintaxis estructuras oracionales tanto perifrásticas como holofrásticas.

\section{Categorías léxicas y clasificación nominal en muinane}

Lyons reconoció que en algunas lenguas la distinción entre nombre, verbo y adjetivo es inaplicable, aunque propuso la distinción nombre versus verbo como universal, ya que ella sí puede ponerse en relación con la distinción independiente y clara entre referencia y predicación. Planteó entonces la necesidad de buscar criterios análogos para examinar las categorías léxicas. Así los nominales son expresiones referenciales desde el punto de vista semántico, mientras que los "adjetivos" considerados como calificativos pueden estar codificados a partir de verbos o de nombres en algunas lenguas. Los adjetivos son definidos como "lexemas" cuya función característica es la de modificar un nombre dentro de un constituyente nominal (Lyons 1977 : 429-452). La escuela funcionalista, por su 
parte, se fundamenta en criterios formales y distribucionales en la diferenciación de las categorías léxicas, atribuyendo prioridad a las funciones prototípicas y no prototípicas de las expresiones y de los lexemas que relacionan la sintaxis y la semántica. Aquí encontramos diversas posiciones sobre la universalidad de los adjetivos como las de Dixon y Aihkenvald (2004). Hopper y Thompson (1984), Givon (1984), Moyse Faurie (2004), y Evans y Osada (2005). De otra parte, investigaciones orientadas a la tipología lingüística como las de Croft (2003, 2005), colocan su acento en los actos proposicionales como son las construcciones referenciales, predicativas y modificativas. Estudios recientes evidencian que la discusión sobre las categorías léxicas en las lenguas del mundo sigue siendo relevante. Lois y Varpnasky (2006) analizan ciertas raíces léxicas como poli categoriales y consideran que la asignación categorial puede darse en la fonología o en la morfosintaxis dependiendo de la lengua. Otros tipólogos observan las categorías léxicas a partir de la distribución dentro del enunciado y la variación de la estructura interna de la palabra en relación con su cohesión y con las demás unidades del mismo. Creissels (2006:15-16).

Para el muinane hemos puesto en práctica la combinación de criterios formales como los fonológicos, morfológicos, sintácticos y discursivos, y planteamos las categorías léxicas nombre y verbo. Una categoría adjetival es difícil de establecer para esta lengua por cuanto no la encontramos como unidad autónoma léxica en función modificadora de los nombres. En la morfología de los nombres compuestos podemos observar nociones calificativas. Sin embargo, esos morfemas hacen parte de un nuevo lexema, casos que ejemplarizamos en (20), (21) y (22).

Antes de entrar en materia mostraremos de manera más concreta algunas características generales de la lengua. En primer lugar sobre la fonología y posteriormente sobre la sintaxis y la clasificación nominal.

\section{Fonología}

La estructura segmental se compone de dieciséis unidades consonánticas y seis vocálicas. La estructura suprasegmental presenta, entre otros, un sistema tonal combinado que describimos en los términos propuestos por (Hyman 2010: 376) entre oposición equipolente y sistema privativo del tono del tipo /A/versus /B/ versus /Ø/, es decir: alto versus bajo versus carencia de tono. El nivel de asignación tonal es el morfema léxico y en pocos casos el morfema gramatical. Veamos en (1) y (2) las melodías de lexemas del lado nominal (tomado de: Vengoechea 2012: 79, 80; tablas (36) y (37)) $)^{2}$

\footnotetext{
${ }^{2}$ Mi traducción del francés al español.
} 
(1) Lexemas nominales

\begin{tabular}{|c|c|c|c|c|c|c|}
\hline Ítem & & $\begin{array}{c}\text { Lexemas } \\
\text { nominales }\end{array}$ & Español & Ítem & $\begin{array}{c}\text { Lexemas } \\
\text { nominales }\end{array}$ & Español \\
\hline & $\begin{array}{l}\text { Melodías } \\
\text { tonales }\end{array}$ & $\mathrm{H} \varnothing$ & & & HB Ø & \\
\hline a. & & búgu & $\begin{array}{l}\text { hacha de madera } \\
\text { de granadillo }\end{array}$ & c. & tû:?e & tapir \\
\hline \multirow[t]{2}{*}{ b. } & & gí:si & piedra & d. & tâ: $\beta a$ & pez \\
\hline & $\begin{array}{l}\text { Melodías } \\
\text { tonales }\end{array}$ & $\mathrm{H}$ & & & $\mathrm{HB}$ & \\
\hline e. & & té:- & quebrada & g. & jgấ- & hamaca \\
\hline f. & & nú:- & materia gris & h. & hư:- & liana \\
\hline
\end{tabular}

Del lado nominal no encontramos jamás una melodía constituida exclusivamente por un tono B. Sin embargo encontramos palabras nominales especificadas para tono $\mathrm{H}$ así como para tono $\mathrm{HB}$.

Los lexemas verbales no están especificados a nivel tonal pues ellos pueden ostentar todas las melodías y ello depende de las categorías

gramaticales o flexiones que realizan:

(2) Lexemas verbales

\begin{tabular}{l|lll}
\hline & $\begin{array}{l}\text { Lexema verbal } \\
+ \text { morfotono }^{3}\end{array}$ & $\begin{array}{l}\text { Lexema verbal } \\
+ \text { morfotono }\end{array}$ & $\begin{array}{l}\text { Lexema verbal } \\
+ \text { morfotono }\end{array}$ \\
\hline $\begin{array}{l}\text { Melodías } \\
\text { tonales }\end{array}$ & B Ø & HB Ø & $\mathrm{H} \varnothing$ \\
& $\begin{array}{l}\text { mùdu } \\
\text { morfotono b : } \\
\text { imperativo-comer. } \\
\text { cacería o pesca } \\
\text { Coma (cacería o pesca) } \\
\text { ! (imperativo) }\end{array}$ & $\begin{array}{l}\text { imperativo- } \\
\text { morfotono b : }\end{array}$ & $\begin{array}{l}\text { múdu } \\
\text { morfotono h : } \\
\text { obligativo- comer. } \\
\text { cacería o pesca }\end{array}$ \\
\hline
\end{tabular}

\section{Sintaxis}

En esta parte describimos brevemente la alineación de los argumentos gobernados por los verbos en muinane. Usamos la propuesta de Comrie (1989 :

${ }^{3}$ Utilizamos la expresión "morfotono" para indicar un tono morfológico. 
104-137), quien propone una definición abstracta y prototípica para el sujeto, mostrando que para las lenguas del mundo podríamos observar dos tipos del mismo. El primer tipo de sujeto es el que codifica de la misma forma el sujeto agente $\mathrm{S}$, A en oraciones intransitivas y transitivas. Una predicación que utiliza este tipo de sujeto es identificada por Comrie con un alineamiento nominativo acusativo de sus argumentos.

El muinane despliega dos tipos de estructuras para las oraciones simples. De hecho, todas las oraciones declarativas de la lengua podrían potencialmente expresarse a partir de dos tipos estructura en una especie de diátesis que hemos identificado con la predicación de tipo 1 (P1) y la predicación de tipo 2 (P2). La primera estructura es perifrástica, o constituida por dos o más términos o palabras (ejs., (3), (4), (5)). En las oraciones de tipo perifrástico el orden más observado antepone frecuentemente el objeto al verbo: (S)OV. El alineamiento es nominativo acusativo en oraciones perifrásticas:

(3) dî:-bô: gâ:se-?i

3P-CL : masc.NOM llegar-P1

Él (macho, hombre) llegó.

(4)

$$
\text { N, PRO. Sujeto Agente V-P1 }
$$

jgá:фi jgàká?ù?i ${ }^{\mathrm{j}} \phi \hat{a}^{\mathrm{j}} \phi \mathrm{a}^{\mathrm{P}}$ bàko

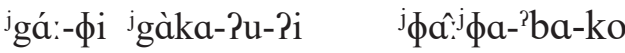

animado-CL : individuo matar-PRET-P1 jabalí-CL : denso.masivo-ACC

Él mató el jabalí.

N.Objeto Paciente

\author{
V-P2 ${ }^{4}$ N.Objeto Paciente \\ jgàkápû:bo $\phi a^{j} \phi a^{2} b a ̀ k o$ \\ jgàká?u-bô: $\quad \phi a^{j} \phi a-{ }^{2} b a-k o$ \\ matar-P2.CL : masc jabalí-CL : denso.masivo-ACC \\ (Él) mató el jabalí.
}

El segundo tipo de sujeto planteado por Comrie (1989 : 104-137) es aquél que codifica de la misma manera el $\mathrm{S}$ de las oraciones intransitivas y el $\mathrm{P}$ paciente de las transitivas en un alineamiento que propone como ergativo absolutivo. Un buen número de lingüistas ha continuado trabajando en esta hipótesis. Creissels (2001: 173-232) analiza el alineamiento de los argumentos

${ }^{4}$ Con P-2 no es necesaria la expresión del pronombre o nombre ya que dentro del verbo tenemos un índice para el sujeto. 
en oraciones de diferentes estructuras. Ahora veamos el alineamiento ergativo absolutivo (a nivel de los índices) para las oraciones holofrásticas en las cuales en muinane tenemos índices del sujeto de la oración intransitiva (cf. (6), (7), (9)) e índices del paciente en las transitivas. Los índices de sujeto y paciente se codifican de la misma manera (cf. (8) y (10)), siempre usando para la tercera persona las marcas de clase nominal, que aquí muestran función argumental en la predicación:

(6) V-P2

(6) gásê:be

gâ:se-bô:

llegar-CL : masc

(Él) llegó.

(7) gá:sége

gâ:se-go

bailar-P2.CL : fem

La (mujer) baila.

La (mujer) está bailando.

(8) má'gákáùkû:bo

mV-jgàka-Pu-kû:-bô:

1/2PL.ERG-apalear-PRET-ACCOM-P2.CL : masc.ABS

Nosotros lo apaleamos (a un hombre, un macho).

(9) ?òhú ${ }^{2} b a$

?òhu- ${ }^{2} \mathrm{ba}$

sonar-P2.CL : denso.masivo.ABS

El (tambor) suena.

(10) mà?ámukû:?ba

mV-Pàmu-kû:-'ba

1/2PL.ERG-tocar-CL : denso.masivo.ABS

Nosotros lo golpeamos (al tambor).

Como indicamos arriba, en muinane, gran parte de las oraciones pueden ser expresadas sin necesidad de aparición del constituyente no predicativo. Aquéllas pueden estar construidas incluso por un único término que indexa a los participantes de la relación predicativa dentro de su estructura. Observamos que cuando el sujeto y el paciente son de tercera persona, tanto la posición como la forma de la 3P dentro de la oración son idénticas, es decir, observamos un tratamiento del sujeto del tipo S, P. En estos casos de tercera persona en 
oraciones holofrásticas observamos alineamiento de tipo ergativo absolutivo que se evidencia por el uso de índices argumentales a partir de las marcas, que en el lado nominal, son marcas de clasificación nominal. Estas oraciones se usan corrientemente. El alineamiento se propuso en Vengoechea (2012: 161, 162) por primera vez, para las lenguas de la familia Bora.

\section{Sistema de clasificación nominal}

Otra forma de caracterizar la lengua muinane en cuanto a la sintaxis son las funciones de su sistema de clasificación nominal. Este sistema puede estar presente en varios puntos del enunciado. De una parte, los marcadores del sistema de clasificación nominal aparecen en el constituyente no predicativo de las oraciones perifrásticas donde es obligatorio con los pronombres de persona como en (3) y (4). De otra parte, los marcadores de clasificación nominal aparecen en el constituyente predicativo de las oraciones holofrásticas. En estas oraciones los marcadores de clase nominal actúan como índices argumentales.

En la identificación y descripción del sistema de clasificación nominal del muinane examinamos los criterios propuestos por Grinevald (2000 : 50-92). El primero es la localización de los morfemas de clasificación nominal. Éstos se sitúan en diversos puntos del enunciado y hacen parte de la formación del constituyente no predicativo dentro de la estructura de nombres, pronombres y numerales. Cuando los marcadores de clase nominal se sitúan en el constituyente predicativo, los encontramos en palabras verbales, adverbiales o nominales en función de objeto. El segundo criterio de Grinevald propone una revisión del inventario del sistema. El muinane muestra un sistema complejo, posee un total de 28 marcadores monosílabos y una serie amplia y productiva de repetidores bisílabos. Además la lengua propone un conjunto de " términos de clase » y un tercer conjunto de « términos de medida ». Éstos tienen la facultad de construir un buen número de palabras compuestas. El tercer criterio de Grinevald nos lleva a estudiar cuidadosamente la semántica de los sistemas de clasificación nominal. El muinane mostrará marcadores con valores semánticos de animado e inanimado. Las marcas de animados se usan exclusivamente para esta clase. Una de las funciones a nivel de la semántica de los marcadores de clase es la de especificar las nociones abstractas expresadas por los lexemas. Ya sea individualizando colectivos, grupos de parentela, hordas, o bien, expresando nociones como: el macho, la hembra, el infante o el animado. Otras marcas usadas para inanimados, y en algunos casos también para animados, se emplean para especificar valores relacionados con la forma, la consistencia, la configuración, la función o el estado del referente designado. Finalmente la lengua propone un marcador monosílabo, desprovisto de semántica, neutro. El último criterio 
propuesto por Grinevald es poner a prueba la vitalidad del sistema. En este sentido el muinane muestra alto grado de vitalidad (cf. Vengoechea 2012: 165170, (22), (23)). A los criterios de Grinevald podemos adicionar uno que plantea describir también la relación existente entre el sistema de clasificación nominal y el léxico que hemos explorado a lo largo de esta propuesta.

Con relación a las reglas de concordancia del sistema de clasificación nominal muinane, ellas son utilizadas como un fenómeno estrictamente discursivo para establecer el grado de relevancia de una característica del referente. Las marcas muestran uso gramatical en muinane exclusivamente como índices argumentales en oraciones holofrásticas.

(11) Marcadores de clase nominal muinane ${ }^{5}$ para animados

\begin{tabular}{|c|c|c|c|c|}
\hline $\begin{array}{c}\text { Marcador } \\
\text { no. }\end{array}$ & Marcador & Semántica & Muinane & Español \\
\hline & & $\begin{array}{l}\text { animados, } \\
\text { noción } \\
\text { específica, } \\
\text { individualizada }\end{array}$ & & \\
\hline 1. & $-2 a$ & $\begin{array}{l}\text { colectivo } \\
\text { humano }\end{array}$ & 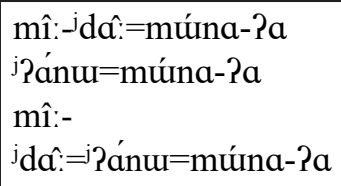 & $\begin{array}{l}\text { los amerindios }{ }^{6} \\
\text { los blancos / } \\
\text { mestizos }^{7} \\
\text { los europeos }\end{array}$ \\
\hline 2. & -ho & $\begin{array}{l}\text { parentela } \\
\text { identidad }\end{array}$ & $\begin{array}{l}{ }^{j} \text { gấ-go-mu-ho } \\
\text { jtû:mo-ho } \\
\text { néhe-jga-mu-ho }\end{array}$ & $\begin{array}{l}\text { la parentela de la } \\
\text { gente de mujeres } \\
\text { la parentela de la } \\
\text { gente gusano } \\
\text { la parentela de la } \\
\text { gente de coco }\end{array}$ \\
\hline 3. & -to & parentela/grupo $^{8}$ & jta-dô:- to & mi familia \\
\hline 4. & $-\mathrm{mo}$ & grupo & hû:si-mo & $\begin{array}{l}\text { consejo de } \\
\text { ancianos }\end{array}$ \\
\hline
\end{tabular}

${ }^{5}$ Los marcadores pueden sufijarse en nombres, verbos, demostrativos, anafóricos y numerales.

${ }^{6}$ La palabra mî.-jdâ: $=$ múna-Pa es un compuesto construido a partir de un morfema de origen verbal y otro nominal. El morfema -múna- jamás aparece sólo *múna, ni tampoco aparece sólo con el clasificador *múna?a. El morfema en cuestión siempre aparece en formas compuestas.

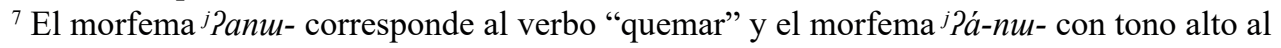
nombre para "ave de rapiña".

${ }^{8}$ Marcador utilizado exclusivamente en palabras del parentesco y palabras gramaticales como los demostrativos o los pronombres. 
12) Marcadores de clase nominal muinane para inanimados con uso en animados:

\begin{tabular}{|c|c|c|c|c|}
\hline Marcador & Marcador & Semántica & Muinane & español \\
\hline & & forma & & \\
\hline 9. & - -?o & $\begin{array}{l}\text { a. largo, rígido } \\
\text { b. enrollado }\end{array}$ & $\begin{array}{l}\text { Púmo-?o } \\
\text { tốka-?o } \\
\text { káha-?o }\end{array}$ & $\begin{array}{l}\text { árbol, planta } \\
\text { serpiente, vr. } \\
\text { verrugosa } \\
\text { árbol caucho }\end{array}$ \\
\hline 10. & $-2 \mathrm{i}$ & \begin{tabular}{|l} 
filiforme \\
suspendido \\
flexible \\
\end{tabular} & $\begin{array}{l}\text { mû:ni-Pî: } \\
\text { Píno-P̂̂: }\end{array}$ & $\begin{array}{l}\text { gusano, vr. } \\
\text { clase de palma }\end{array}$ \\
\hline 11. & $-P u$ & protuberante & $\begin{array}{l}\text { jtú:du-Pu } \\
\text { bá:da-?u }\end{array}$ & $\begin{array}{l}\text { golondrina } \\
\text { pez, sp., botello }\end{array}$ \\
\hline 12. & $-j$ Pa & $\begin{array}{l}\text { convexo } \\
\text { redondeado }\end{array}$ & $\begin{array}{l}\text { hû́:mu-jípa } \\
\text { Píno-ji?a }\end{array}$ & $\begin{array}{l}\text { mico, vr. tanque } \\
\text { guacamaya, vr. } \\
\text { canangucho }\end{array}$ \\
\hline 13. & -ga & $\begin{array}{l}\text { convexo } \\
\text { aplanado }\end{array}$ & $\begin{array}{l}\text { táta-ga } \\
\text { ?íka-ga }\end{array}$ & $\begin{array}{l}\text { tierra vr., blanca, } \\
\text { no fértil }\end{array}$ \\
\hline 14. & - the & $\begin{array}{l}\text { circunferencia, } \\
\text { plano }\end{array}$ & 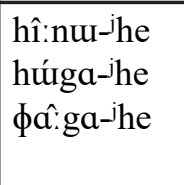 & $\begin{array}{l}\text { planeta tierra } \\
\text { hacha (en metal } \\
\text { ou madera) } \\
\text { araña, vr. }\end{array}$ \\
\hline \multirow[t]{2}{*}{15.} & -hw & cilindro & $\begin{array}{l}\text { mê:ne-hu } \\
\text { Pìmi-hu } \\
\text { tû:si-hu }\end{array}$ & $\begin{array}{l}\text { camino } \\
\text { buenas palabras } \\
\text { fruto del guamo }\end{array}$ \\
\hline & & consistencia & & \\
\hline 16. & $--^{2} \mathrm{ba}$ & $\begin{array}{l}\text { denso } \\
\text { masivo }\end{array}$ & $\begin{array}{l}\text { nô:--'ga-ºba } \\
\text { hî:nu-'ba } \\
\text { húgo-'ba } \\
\text { káha-'ba }\end{array}$ & $\begin{array}{l}\text { gravilla } \\
\text { barro } \\
\text { jarro } \\
\text { fruto del caucho }\end{array}$ \\
\hline \multirow[t]{2}{*}{17.} & $-j \phi a$ & acuoso & $\begin{array}{l}\text { nî:-joa } \\
\text { nô:- }{ }^{2} b a-{ }^{j} \phi a\end{array}$ & $\begin{array}{l}\text { gusano, sp. } \\
\text { serpiente coral }\end{array}$ \\
\hline & & configuración & & \\
\hline 18. & -Pu & enrollado & $\begin{array}{l}\text { pû:nu-Pu } \\
\text { nî:ga-Pu } \\
\text { sû:ta-?u }\end{array}$ & $\begin{array}{l}\text { araña } \\
\text { cabeza } \\
\text { cigarra }\end{array}$ \\
\hline 19. & $-\mathrm{b} \mathrm{ba}$ & $\begin{array}{l}\text { vertebrado, } \\
\text { tejido }\end{array}$ & $\begin{array}{l}\text { hûi:?i-jba } \\
{ }^{j} \text { dô:-jba }\end{array}$ & $\begin{array}{l}\text { canasto } \\
\text { columna vertebral }\end{array}$ \\
\hline
\end{tabular}




\begin{tabular}{|c|c|c|c|c|}
\hline 20. & -jga & ramificado & $\begin{array}{l}\text { Púse-jga } \\
\text { káha-jga }\end{array}$ & $\begin{array}{l}\text { dedo de la mano } \\
\text { semillas de } \\
\text { caucho }\end{array}$ \\
\hline \multirow[t]{2}{*}{21.} & $-\mathrm{mi}$ & $\begin{array}{l}\text { colectivo } \\
\text { conjunto de } \\
\text { objetos idénticos }\end{array}$ & kû:me-mi & $\begin{array}{l}\text { palmeral sp. } \\
\text { milpesos }\end{array}$ \\
\hline & & facultad & & \\
\hline 22. & $-d a$ & brillante & $\begin{array}{l}\text { mê:ku-da } \\
\text { dê:-da } \\
\text { hố-da } \\
\phi \hat{a}-d a \\
\text { mâ:-da }\end{array}$ & $\begin{array}{l}\text { estrella } \\
\text { boa de fuego } \\
\text { yuca dulce } \\
\text { acto sexual } \\
\text { esperma }\end{array}$ \\
\hline 23. & -jma & alegre & kàduu=dàna-ja $m a$ & canción \\
\hline 24. & ${ }_{-j}^{j} \mathrm{duu}$ & acústico $^{9}$ & ใô:hu- ${ }^{-j} \mathrm{duu}$ & glotis \\
\hline 25. & $-\mathrm{ku}$ & $\begin{array}{l}\text { peligroso } \\
\text { depredador } \\
\text { redondo }\end{array}$ & $\begin{array}{l}\text { dí:si-ku } \\
\text { tû:gi-ku }\end{array}$ & $\begin{array}{l}\text { pozo profundo } \\
\text { pez, sp. Mojarra }\end{array}$ \\
\hline 26. & -ha & abrigo & ká:mo-ha & casa o abrigo alta \\
\hline 27. & $-\mathrm{j} \mathrm{d} \hat{\mathrm{i}}$ & $\begin{array}{l}\text { pertenencia } \\
\text { (en el sentido } \\
\text { cultural) } \\
\text { propiedad } \\
\text { (característica) }\end{array}$ & hô- jâ: & $\begin{array}{l}\text { palma, sp. } \\
\text { bombona }\end{array}$ \\
\hline 28. & - no & neutro $^{10}$ & 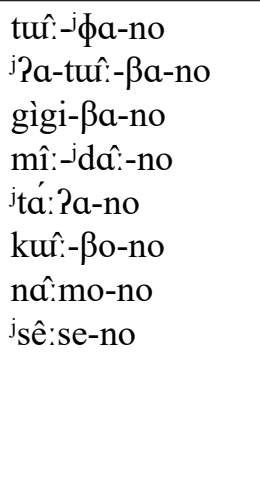 & $\begin{array}{l}\text { ser rojo } \\
\text { ser verde/azul } \\
\text { ser amarillo } \\
\text { ser verdadero, } \\
\text { propio } \\
\text { ser falso } \\
\text { ser oscuro } \\
\text { ser dulce, ser } \\
\text { claro }\end{array}$ \\
\hline
\end{tabular}

${ }^{9}$ El clasificador ${ }^{j} d u$, caracterizado como "acústico" se sufija a morfemas nominales completando el contenido de la referencia, indicando que se trata de un objeto con capacidad acústica como "glotis".

${ }^{10}$ Las nociones que afijan el clasificador neutro son por lo general verbos dentro del constituyente predicativo. También puede ser usado en el constituyente no predicativo por pronombres o demostrativos y verbos nominalizados. 
(13) Muestra de términos de clase, de términos de medida y clases nominales bisílabas:

\begin{tabular}{|c|c|c|c|c|}
\hline $\begin{array}{l}\text { Término } \\
\text { no. }\end{array}$ & Término & Semántica & Muinane & Español \\
\hline \multirow[t]{2}{*}{1.} & -húku & Jaguar & kâ:ha-?o-húku & jaguar de caucho \\
\hline & & & kúme-Pi-húku & $\begin{array}{l}\text { palma de jaguar, sp. } \\
\text { milpesillo }\end{array}$ \\
\hline \multirow[t]{2}{*}{2.} & -?â:me & Hoja & kâ:ha-?o-?â:me & hoja del árbol de caucho \\
\hline & & & tû̂:si-Po-?â:me & hoja del árbol de guamo \\
\hline \multirow[t]{2}{*}{3.} & -mê:?u & corteza & kúmu-ku-mê:?u & liana curva, sp. mono \\
\hline & & & gâ:da-mê:?u & liane curva sp. \\
\hline \multirow[t]{2}{*}{4.} & -tí:-jdu- & bulto & 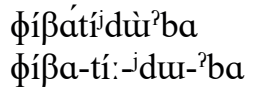 & $\begin{array}{l}\text { bulto de objetos } \\
\text { diversos }\end{array}$ \\
\hline & & & 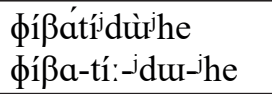 & $\begin{array}{l}\text { bulto de objetos de la } \\
\text { misma calidad }\end{array}$ \\
\hline \multirow[t]{2}{*}{5.} & -dû:si & canasto & $\begin{array}{l}\text { má:Púnódúsi } \\
\text { mâ:Pu-no-dû́:si }\end{array}$ & cnasto de cazabe \\
\hline & & & 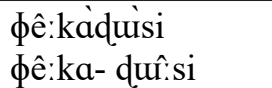 & canasto de raíces \\
\hline 6. & $-g a^{j} d u$ & diminutivo & $\mathrm{j}_{\mathrm{ta}} \hat{\mathrm{d}} \mathrm{di}-\mathrm{ga} \mathrm{a}^{\mathrm{j}} \mathrm{du}$ & abuelo (diminutivo) \\
\hline 7. & -dubi & afectivo & jtâ:di-dubi & abuelo (afectivo) \\
\hline 8. & -Puba & atenuativo & kû::ßo-?uba & ser negruzco \\
\hline 9. & -Pubu & máximo & jtâdi-?ubu & abuelito (finado) \\
\hline 10. & -mấ.jhe & estrategia & dúdứká=mâ. he & estrategia de trabajo \\
\hline
\end{tabular}

Observamos que el sistema muinane, compartido con algunas diferencias por las lenguas de familia lingüística Bora (cf. Seifart 2005), exhibe las mismas características del sistema de clasificación nominal de las lenguas de la familia Tukano oriental en lo que refiere a varias de sus funciones gramaticales y discursivas. La lengua barasana cuenta con cuatro tipos de concordancia en el verbo según Gomez-Imbert (2007).

(14) Lengua barasana, familia Tukano oriental, cuadro en Gomez-Imbert (2007: 60):

Semántica

a. +AN, +PL, nM Ẽacá

b. $+\mathrm{AN},-\mathrm{PL},+\mathrm{M}$ jáí

c. $+\mathrm{AN},-\mathrm{PL},-\mathrm{M}$ jáí-ó

d. $-\mathrm{AN}, \pm \mathrm{PL}, \mathrm{nM}$ kúbú-á verbo barasana

wadí-á-ba

wadí-á-bi

wadí-á-bo

wadí-á-ha

\section{Español}

Las gentes vienen.

Un jaguar viene.

Una perra viene.

Una piroga viene. 
Mientras tanto en muinane podemos observar un sistema de concordancia, para nosotros de tipo argumental, como el siguiente cuando se utiliza el marcador de clase nominal en el verbo. En esta lengua la aparición del constituyente no predicativo no es siempre necesaria.

(15) Lengua muinane

\begin{tabular}{|l|l|l|l|l|}
\hline & \multicolumn{1}{|c|}{ Semántica } & \multicolumn{1}{|c|}{$\begin{array}{c}\text { (Constituyente } \\
\text { no predicativo) } \\
\text { muinane }\end{array}$} & $\begin{array}{c}\text { Constituyente } \\
\text { predicativo } \\
\text { verbo muinane }\end{array}$ & \multicolumn{1}{|c|}{ Español } \\
\hline a. & AN, PL, nM & (mìidámùnài?a) & sá:-mo & Las gentes vienen. \\
\hline b. & AN, SG, M & (húku) & sá:-bô: & El jaguar viene. \\
\hline c. & AN, SG, F & (?'jijgá:gómé:ni) & sá:-go & $\begin{array}{l}\text { La cerrillo (hembra) } \\
\text { viene }\end{array}$ \\
\hline
\end{tabular}

Lo anterior indica que tanto en las lenguas de la familia Bora como en las lenguas de la familia Tukano oriental referidas encontramos sistemas de clasificación nominal cuyos marcadores en el constituyente predicativo muestran la función de indicar el argumento o argumentos de la relación predicativa, específicamente en aquellos casos en los que el constituyente no predicativo no es necesario. Observamos también una similitud en la semántica de los diferentes sistemas que parten en todos los casos de la distinción entre animado e inanimado.

Luego de analizar las características sobresalientes de la lengua en cuanto a su fonología, sintaxis y sistema de clasificación nominal, es posible presentar las categorías léxicas de la lengua y su relación con el sistema. Proponemos dos categorías léxicas en muinane: nombre y verbo. Desde los puntos de vista de la sintaxis y de la predicación, la categoría nombre puede ser definida como aquella unidad del léxico cuya función semántica es la de persona u objeto y cuya función dentro de la predicación es referencial (cf. Lyons 1977 y Croft 2000). Los nombres pueden ser más o menos prototípicos (Croft 2000). Los últimos son unidades léxicas no marcadas. La categoría léxica nombre se construye morfológicamente a partir de un lexema y sus afijos. Aquí exploramos la distinción entre dos tipos de flexión en la formación de las palabras de Booij (1993): la flexión inherente y la flexión contextual, distinción útil dada la complejidad morfológica de la lengua. Los morfemas inherentes en su orden de importancia y obligatoriedad son: el morfema de clase nominal (CL), el prefijo posesivo y uno de los morfemas de número, utilizado eventualmente. Los afijos que muestran una función sintáctica del constituyente son aquéllos que marcan la función casual en la oración. En lo ejemplos en ((16) a (19)) podemos observar nombres que cumplen con los criterios mencionados: 
(16) $\mathrm{N}$ REL

dê:ka PV-ßòsu-kû:-?i

flor REL-ser hermoso-ACCOM-P1

Flores que ya son hermosas.

(17) $\mathrm{N} \quad \mathrm{V}$

sê:me Pùgo-?i

niño caminar-P1

El niño camina.

(18) $\mathrm{N} \quad \mathrm{N}$ V

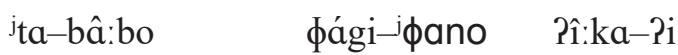

POss1 P-abuela chagra-INES ser.estar.vivir-P1

Mi abuela está en la chagra.

(19) $\mathrm{N} \quad \mathrm{V}$

mî:-jdô:=muína-?a mV-?î:ka-bô:

ser verdadero-CL : pertenencia-gente $\quad$ 1/2PL-ser.estar.vivir-P2.CL: masc

-CL : collectivo.humano

La criatura de los verdaderos hombres somos (nosotros).

En la formación de los lexemas nominales encontramos una serie amplia de compuestos como los que mostramos a continuación. Ninguna de sus partes puede ser considerada en forma de unidad autónoma por razones de prosodia y cohesión de la palabra. Sin embargo, consideramos que es necesario continuar explorando estos ejemplos a nivel de la oración y del discurso, ya que la diferenciación entre compuestos y sintagmas no es siempre evidente en las lenguas. Palabras y sintagmas pueden observar las mismas funciones sintácticas y además los compuestos derivan de sintagmas en muchas lenguas. Uno de los criterios propuestos por los morfólogos para diferenciar entre compuestos y sintagmas es la existencia o inexistencia de morfemas de concordancia (Booij 2007:82). En muinane no planteamos morfemas de concordancia ya que la lengua no obliga a ella. Si hay repetición de algún morfema es más un procedimiento discursivo. Veamos los ejemplos (20) a (22).

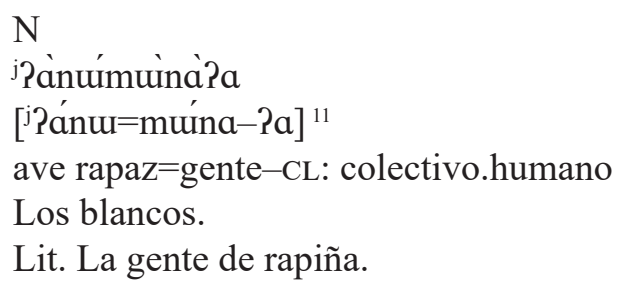

Lit. La gente de rapiña.

${ }^{11}$ Los paréntesis cuadrados indican aquí el límite de palabra. 
(21) Compuesto sintáctico ${ }^{12}$

Páj dù?òmùnà?a

[?ádiu-Po=múna-Pa]

arriba-marcador genitivo=gente-CL:colectivo.humano

Gente de arriba (los uitoto).

(22) $\mathrm{N}$

Pìtúkònuimììàmùnà?a

[?i-túkònu=mî:-idâ:=múna-Pa]

[POSS : $3 \mathrm{P}^{13}$-principio $=$ ser verdadero-CL:pertenencia $=$ gente-CL:colectivo.

humano]

los primeros amerindios

La categoría verbal prototípica corresponde a la unidad léxica que cumple una función predicativa en la relación y su función semántica corresponde a una acción (Croft 2003). En muinane se construye la categoría verbal con un morfema léxico y sus afijos. El verbo muestra sufijos inherentes de tiempo y aspecto. Dentro de los afijos contextuales aquél presenta modalidad, polaridad, marcas discursivas. El único prefijo es el de persona intralocutiva. La tercera persona puede aparecer como sufijo final en la forma de un marcador de clase nominal $\{-\mathrm{CL}\}$ en la palabra verbal, o en su defecto, se sufija en esa posición final una marca predicativa: $\{-\mathrm{Pi}\}$ como en los ejemplos (23) a (25):

(23) PRO V

(?úhó?o) Ø-?ùgo-kû:-7i

(1SG) 1sG-caminar-ACCOM-P1

(Yo) ya caminé.

(24) PRO

$\mathrm{V}$

dî:-bô:

dû:- $\phi i-k u ̂:-? i$

3P-CL: masc comer.proteína.animal-HAB-ACCOM-P1

Él está ya habituado a comer.

(25) $\mathrm{V}$

dû:-фi-kû:-bô:

comer.proteína.animal-HAB-ACCOM-P2.CL:masc/macho

(Él) está ya habituado a comer.

\footnotetext{
${ }^{12}$ El marcador genitival es de uso muy restringido y su construcción representa un compuesto sintáctico.

${ }^{13} \mathrm{La}$ aparición de un prefijo de posesión nominaliza toda la palabra que contiene una noción verbal.
} 
Según Lyons (1977), las nociones adjetivales o calificativas pueden estar codificadas a partir de verbos o de nombres en algunas lenguas del mundo. Para Dixon y Aihkenvald (2004:36), el adjetivo es una categoría léxica universal. Estos autores proponen para las lenguas del mundo clases léxicas adjetivales que expresan nociones de tipo central como: edad, valor, color y nociones de tipo periférico como: talla, forma y propensión humana para los adjetivos. En muinane, revisando estos casos obtenemos siempre verbos, nombres derivados de verbos y oraciones relativas con sujeto explícito en algunos casos o con cabeza interna en otros. Veamos algunos de estos casos en muinane:

\section{Edad}

(26) $\mathrm{N}$

bágòka-ha

joven-CL:abrigo ${ }^{14}$
$\mathrm{N}$ j?a-mî:-jdâ:-go POSS.PAR-ser verdadero-CL:pertenencia CL:fem/hembra ${ }^{15}$
(27) N

фúku-?ba-ko

adorno-CL: denso.masivo-ACC

\section{REL}

?V-фî:ku-no

REL-1levar-P2.CL: neutro

La joven, la vieja, el adorno es lo que llevan.

\section{Valor}

(28) $\mathrm{N}$

híme-?o

pulsera-CL: largo

La pulsera (es) bonita.
$\mathrm{V}$

Pìmi-no

ser bueno/ser bello-CL: neutro

$\mathrm{V}$

Pímììnùno

Pìmi-Pìnu-no

ser bueno/ser bello-REM-CL: neutro

La pulsera (era) bonita.

\footnotetext{
${ }^{14}$ El concepto de una muchacha joven incluye en su semántica también la noción del clasificador - $h a$ el cual se refiere a la facultad de abrigo. Podemos comprender su uso a partir de la concepción cultural de género femenino que implica capacidad de abrigar.

${ }^{15}$ La palabra ${ }^{j} \mathrm{q} \mathrm{a}-m \hat{\imath}^{-}{ }^{j} d \hat{a}:-g o$ para referirse a: "la (mujer) vieja", muestra una morfología diversa. En realidad es la nominalización de una palabra verbal, derivación realizada a partir la prefijación del morfema de posesión parental ${ }^{j}$ ?a- De otra parte, La noción de "ser verdadero" -mî: aparece sufijada por dos morfemas de clase nominal. El primero es ${ }^{j} d \hat{a}$ :que significa pertenencia, identidad. El segundo morfema de clase es -go, que refiere a femenino.
} 
(30) PRO

hí:-?a
$\mathrm{V}$

?ìmi-tu- ${ }^{2} \mathrm{ba}$

ser bueno/ser bello-NEG-CL: denso.masivo

Este fruto no está bueno.

\section{Color}

(31) REL ${ }^{16}$

Tìgígißà̀du

PV-jîi:gi- $\beta a-j \mathrm{~d} u$

REL-Ser amarillo- $-\mathrm{VOZ}_{\text {experimentante }}-\mathrm{P} 2$.CL: acústico

La (cuerda) que es amarilla.

(32) PRO

hí: jou

$\mathrm{V}$

hí:- - du

jgígißàjdu

DEM.C-CL: acústico

Ésta (es) una (cuerda) amarilla.

ser amarillo- $-\mathrm{VOZ}_{\text {experimentante }}-\mathrm{P} 2$.CL: acústico

(33) INT

múi ${ }^{j} d u$

$\mathrm{N}$, derivado

múu ${ }^{j} d u$

Pitứ'i $\phi a$

$\mathrm{V}$

Poss 3

?i-tû: ${ }^{j} \phi a$

hé:ßà?i

¿Como parece la roja?

hê: $\beta a-7 i$

parecer-P1

\section{Talla}

(34) NUM

sá $^{j} d u$

V

sá $-{ }^{\mathrm{j}} \mathrm{duu}$

NUM :1-CL: acústico

gìdá: jidu

gî: $d a-{ }^{j} d u$

Una (cuerda) es la (cuerda) grande.

ser grande-P2.CL: acústico

Forma

(35)
$\mathrm{N}$
?ínìgà?u
N V
Pi-níga-Pu
фáPu Píkàtùno
POSs3P-CL: enrollado
фá?u î̀:ka-tu-no
Bola
vivir.estar.ser-NEG-CL: neutro
Literal: Su cabeza no es una bola.
Su cabeza no es redonda.

\footnotetext{
${ }^{16}$ Oración relativa con cabeza interna.
} 


\section{Propensión humana}

(36)

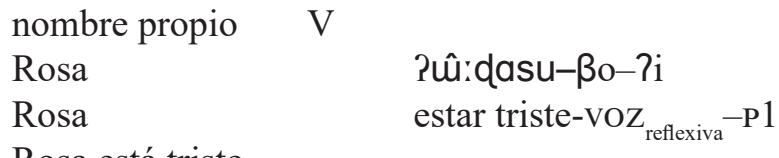

Rosa está triste

En todos los casos anteriores observamos que la codificación de las nociones calificativas en muinane es mixta. Ellas se expresan como verbos, como nombres derivados de verbos o como oraciones relativas. El sistema de clasificación nominal opera en el constituyente no predicativo y en el predicativo. Los marcadores de clasificación ostentan funciones sintácticas y léxicas en los ejemplos mostrados.

\section{Categorías léxicas en lenguas de la familia Bora y su relación con la clasificación nominal}

Las tres lenguas de la familia Bora, como hemos dicho anteriormente, son el bora propiamente dicho, el miraña y el muinane. Seifart (2005: 47) para el miraña, considera que los criterios fonológicos como palatalización y tono sirven para identificar el límite de palabra. Propone criterios morfosintácticos para delimitar las partes del discurso como nombres y verbos. No encuentra adjetivos como una categoría separada y observa necesario profundizar en el tema. Observa la expresión de las propiedades calificativas a partir de oraciones relativas. Para Seifart los adverbios tampoco son una categoría separada (2005: 51). La clasificación nominal del miraña ha sido estudiada ampliamente por este lingüista, quien propuso la función derivacional de los marcadores de clase en los nombres y su uso en las demás partes del discurso y de la predicación. La concordancia de los sistemas de clasificación nominal en distintos puntos del enunciado es planteada como una de las cualidades principales del sistema, la que opera a partir de reglas en miraña, observando además funciones de tipo discursivo. Entre ellas la función anafórica (2005: 105-177). Seifart y Payne (2007) también han trabajado en la tipología de estos sistemas en la región. Los sufijos clasificadores tienen en todas las lenguas estudiadas para el Noroeste amazónico una función derivativa muy productiva en la creación de nuevos nombres. Además ellos aparecen en contextos morfosintácticos adicionales. Para la lengua bora contamos con la propuesta de Thiesen y Weber (1966 y 2001). Estos autores consideran a los "adjetivos" como derivados de los verbos o de los participios. Su semántica, plantean, es la de resultado. Ellos denominan también adjetivos a otras palabras con semántica de propiedad, lo que hace 
pensar que se refieren a nociones adjetivales, ya que al mismo tiempo afirman no haber encontrado palabras adjetivales en función modificadora de un nombre dentro de los constituyentes de la lengua (Thiesen y Weber 2001: 87 y 185).

\section{Otras lenguas de la región amazónica, la lengua andoque y lenguas de las familias Uitoto, Tukano oriental y centro oriental}

La lengua uitoto estudiada en una de sus variedades por Petersen de Piñeres (1994) exhibe categorías nominales y verbales. Las nociones típicas de los adjetivos son codificadas en forma de verbos o de nombres. La lengua andoque descrita por Landaburu y hablada en la región, presenta radicales nominales y verbales (Landaburu 2011: 1571). Hay un buen número de lenguas de la familia Tukano oriental y por esta razón me refiero únicamente a las lenguas tatuyo, y barasana. En la Morphologie et phonologie barasana: approche non linéaire Gómez-Imbert (1997) no reporta la existencia de adjetivos como unidades léxicas autónomas. Las unidades léxicas que se deducen a partir de su morfología son nombres y verbos. En tatuyo tampoco se reporta de parte de la misma investigadora la categoría adjetival como unidad autónoma (GómezImbert 1982). La lingüista identifica dos clases de lexemas: nombre y verbo. Las nociones adjetivales se expresan a partir de verbos (Gomez-Imbert 2011: 1559). En la lengua tukano propiamente dicha, Ramírez (1997) reporta nombres y verbos. Las dos categorías son subdivididas en autónomas y dependientes. Las nociones calificativas son para este autor verbos dependientes. Para la familia Tukano centro oriental, lenguas como el siriano, descrito por Brandrup y Criswell (2000: 400) como muy cercana del desano, muestran la relevancia del verbo como único elemento obligatorio de la predicación, excepto cuando hay cambio de sujeto. En cuanto a las nociones adjetivales, ellas aparecen en algunos ejemplos que el autor presenta, siempre sufijadas con lo que el llama un "sustantivizador". De otra parte, el siriano posee también un rico sistema de clasificación nominal. Todo lo anterior nos hace pensar en un conjunto de rasgos de las lenguas del área geográfica que nos interesa aquí. Entre ellos, la carencia de la clase adjetival como unidad autónoma modificadora de los nombres en lenguas que precisamente despliegan sistemas complejos y omnipresentes de clasificación nominal, rasgos que puede estar en relación con otros aspectos de la tipología de estas lenguas.

\section{Consideraciones antropológicas y lingüísticas finales}

Las sociedades tradicionales que habitan la Amazonía colombiana han sufrido en el último siglo procesos de cambio cultural y lingüístico considerable. Se ha dado una recomposición poblacional de grupos étnicos, clánicos y 
familiares debido a la inserción de estas poblaciones en las prácticas económicas foráneas de extracción de recursos naturales, así como a los conflictos sociales y armados que han azotado la región. Las lenguas probablemente han cambiado. Observamos una serie de coincidencias tipológicas en un grupo areal de lenguas de las familias Bora, Tukano oriental, Tukano centro oriental, Uitoto y en la lengua andoke. De una parte estas lenguas muestran dos categorías léxicas: nombre y verbo. Las referidas lenguas codifican las nociones de propiedad y cualidad a partir de una de estas dos clases léxicas. Algunas de las lenguas estudiadas cuentan además con una clase adverbial. Todas poseen sistemas complejos y omnipresentes de clasificación nominal. Estos sistemas se expresan tanto en las categorías del léxico como en las demás partes del discurso. En la categoría nominal se observan casos en los que el marcador de clase se usa como un morfema léxico. En su mayor parte los marcadores operan como morfemas cuya función derivacional cambia el significado del morfema léxico de base. Por escogencia de una estructura oracional holofrástica de parte del hablante, los marcadores de clase pueden actuar como índices argumentales de tercera persona y portar funciones sintácticas dentro la categoría verbal y la oración. No hay ningún tipo de concordancia obligatoria. El sistema exhibe además varias funciones discursivas. Una de las funciones discursivas que proponemos nosotros es la de saliencia. Se hace entonces relevante una característica específica del referente (femineidad, peligrosidad, redondez, abrigo, etc.,) dentro del discurso. Estas nociones se convierten incluso en rastreadores del referente. Es aquí donde planteamos por primera vez para estas lenguas una relación entre la carencia de una categoría sintáctica adjetival y la presencia de sistemas de clasificación nominal con funciones en la morfología, la sintaxis, el léxico y el discurso. Por lo expuesto aquí podemos concluir que los sistemas de clasificación nominal en las lenguas amazónicas mencionadas operan no sólo en la construcción interna de los constituyentes y de la oración misma, sino también en los niveles léxico, sintáctico semántico y discursivo. Se requiere de mayor exploración e investigación en las lenguas de la región en los aspectos menos conocidos de los sistemas de clasificación nominal y su relación con el léxico.

\section{Abreviaturas y convenciones}

ABS: absolutivo. AG: agente. A: tono alto. ACC: acusativo. ACCOM: cumplido. AG: agentivo. AN: animado. ASP: aspecto. B: tono bajo. C: consonante. CAUS: causativo. CL: clase nominal. DEM: demostrativo. DEM.C: demostrativo cercano. DEM.D: demostrativo distal. DIR: direccional. EXORT: exhortativo. fem: femenino/hembra. H: tono alto. ERG: ergativo. IMP: imperativo. IMPERF: imperfectivo. IN: inanimado. INES: inesivo. INT: interrogativo. 
IPFV: imperfectivo. masc: masculino/macho. MOD: modalidad. N: nombre. NEG: negación. nM: no masculino. NUM: numeral. P: persona. P1: predicación de la clase 1. P2: predicación de la clase 2. PFV: perfectivo. PL: plural. POL: polaridad. POSS: posesivo. POSS.PAR: posesivo parental. PRET: pretérito. PRO: pronombre. PROS: prospectivo. REL: relativa/relativo. REM: remoto. RESUL: resultado. SG: singular. SOC: sociativo. sp.: especie. SUJ: sujeto. V: verbo. VIRT: virtual. vr.: variedad.

( ): opcional. [ ]: límite de palabra compuesta. = unión de dos lexemas en una palabra compuesta. Ø: ausencia de tono en fonología, morfema cero en morfología.

\section{Bibliografía}

Aikhenvald, Alexandra. "Unusual Classifiers in Tariana”. En: Senft, G. Ed. 93-113, 2000.

Bandrup, Beverly y Linda, Criswell. "Un bosquejo fonológico y gramatical del siriano." En: González de Pérez y Rodríguez de Montes eds. 395-417, 2000.

Bonvini, Emilio, Busutil, Joëlle, y Alain, Peyraube eds. Dictionnaire des Langues. Paris: Presses Universitaires de France, 2011.

Booij, Geert. "Inherent versus contextual morphology in the splits morphology hypothesis." 1993. Referencia en la red, consultada en agosto 2012. http://www. hum2.leidenuniv.nl/booijge/pdf/.

Comrie, Bernard. Language Universals \& Linguistic Typology. Segunda Edición. Chicago : The University of Chicago Press. 1989.

Creissels, Denis. Syntaxe générale. Une introduction typologique. Paris : Lavoisier, 2006.

Croft, William. "Parts of Speech as Language Universals and as Language Particular Categories". En: Vogel y Comrie. 65-102, 2000.

Croft, William. Typology and Universals. Segunda edición. Cambridge: Cambridge University Press, 2003.

Croft, William. "Word Classes, Parts of Speech, and Syntactic Argumentation". Linguistic Typology. 9 (3). 431-441. 2005.

Dixon, Richard, M., W. y Alexandra, Aikhenvald. Adjective Classes. A CrossLinguistic Typology. Oxford : Oxford University Press, 2004.

Evans, Nicholas y Toshiki, Osada, "The Myth of a Language without Word Classes". Linguistic Typology. 9 (3) : 351-390, 2005.

Givon, Talmy. Syntax. An Introduction. V.I. Amsterdam/Philadelphia : John Benjamins, 2001. 
Gómez-Imbert, Elsa. “Le Tatuyo”. En: Bonvini, E., Busutil, J., y A., Peyraube eds. Resp. sur les langues d'Amérique : F. Queixalós. 1454-1460, 2011.

Gómez-Imbert, Elsa. Morphologie et phonologie Barasana : Approche nonlinéaire. Doctorat d'État sous la direction de François Dell. Université de Paris 8. UFR : 6 Sciences du Langage. Manuscript, 2007.

Gómez-Imbert, Elsa. "Nominal Classification in Tukanoan Languages". En: Leo Wetzels ed. 401-429, 2006.

Gómez-Imbert, Elsa. De la forme et du sens dans la classification nominale en Tatuyo. (Langue tukano orientale d'Amazonie colombienne). Dissertation. TDM 19. Paris. Université de la Sorbonne Paris IV. Paris : Ed. ORSTOM, 1982.

González de Pérez María Stella, y Rodríguez de Montes María Luisa, eds. Lenguas indígenas de Colombia: una visión descriptiva. Bogotá: Instituto Caro y Cuervo, 2000 .

Grinevald, Colette. "A Morphosyntactic Typology of Classifiers." En: Senft, Gunter, Ed. 50-92, 2000.

Hyman, Larry M. "Amazonia and the typology of tone systems." University of Berkeley. Phonology Lab Annual Report 2010. Presentado en Amazónicas III. Sometido para publicación en el volumen Laryngeal Features, Wetzels, Leo; Gomez-Imbert, Elsa y Bartolomé Pacheco eds. Referencia en la red. Consultada 08.04.2014. http://linguistics.berkeley.edu/phonlab/annual_report/annual_ report_2010.html.

Landaburu, Jon. “L'Andoke” En: Bonvini, Emilio, Busutil, Joëlle, y Alain, Peyraube eds. Resp. sur les langues d'Amérique, 2011.

Landaburu, Jon. "Clasificación de las lenguas indígenas de Colombia”. En: González de Pérez María Stella y Rodríguez de Montes María Luisa, eds. 2548, 2000.

Lois, Ximena y Valentina Vapnarsky. Eds. Lexical Categories and Root Classes in Amerindian Languages. Bern: Peter Lang, 2006.

Lyons, John. Semantics. V 1 y V 2. Cambridge: Cambridge University Press, 1977.

Moyse-Faurie, Claire. Catégorisation lexicale et problème de l'opposition verbo nominal. Mémoire en vue d'obtention de l'habilitation à diriger des recherches, dirigé par Alain Lemaréchal, Habilitation soutenue à Paris IV. Université de Paris, 2004.

Orjuela, Lorena y Ospina, Ana María. "Juegos para la elicitación de datos sobre propiedades, estados, procesos y acciones." Experimentos del Proyecto: Tipología fonológica y gramatical de las lenguas amerindias y andinas de Colombia. ECOS-NORD. Manuscrito, 2010.

Petersen de Piñeres, Gabrielle. "Nominal Clasification in Uitoto". International Journal of American Linguistics. 73 (4) : 389-409, 2007. 
Payne, Doris L. "Source of the Yagua Nominal Classification." International Journal of American Linguistics. 73 (4) : 447-474, 2007.

Ramirez, Henri. A Fala Tukano dos YE'PA-MASA. T.I. Manaos: Cedem, 1997.

Seifart, Frank. The Structure and Use of Shaped-based Noun Classes in Miraña (North West Amazon). WageningIn : Ponsen y Looijen, 2005.

Seifart, Frank. "The Prehistory of Nominal Classification in Witotoan Languages." International Journal of American Linguistics. 4: 411-445, 2007.

Seifart, Frank y Payne, Doris. "Nominal Classification in the North West Amazon: Issues in Areal Difusion and Typological Characterization." International Journal of American Linguistics. 73 (4): 389-409, 2007.

Senft, Gunter. Systems of Nominal Classification. Cambridge: Cambridge University Press, 2000.

Thiesen, Wesley y David, Weber. A Grammar of Bora. Manuscrito, 2001.

Thiesen, Wesley. Gramática del idioma bora. Wise, M. R., ed. Lima : Ministerio Educación Perú, y SIL, 1996.

Vengoechea, Consuelo. "Muinane y su sistema de clasificacion nominal: interacción entre sintaxis y pragmática." Seminario CLANLINC: La Clasificación Nominal en las Lenguas Amazónicas. Laboratoire Dynamique du Langage. Lyon: Universidad de Lyon 2. Inédito. 2001.

Vengoechea, Consuelo. “Análisis del nombre a partir del léxico del cuerpo humano en muinane.” Forma y Función. 16. 285-291, 2003.

Vengoechea, Consuelo. "Morphosyntax of Muinane.” Amerindia. 29/30 : 119-140, 2004-2005.

Vengoechea, Consuelo. Catégorisation lexicale en Muinane. Amazonie colombienne. Tesis doctoral. Universidad de Toulouse Le Mirail. Francia. Directores: Investigadora C.N.R.S., Doctora de Estado: Elsa Gomez-Imbert y Profesor Investigador Universidad de Toulouse: Dr. Jacques Durand, miembro del Instituto Universitario de Francia. Sustentada 2012. Referencia en la red, consultada 01.04.2014. http://tel.archives-ouvertes.fr/docs/00/79/67/60/PDF/ De_-_Vengoechea_Consuelo.pdf.

Vogel, Petra, M., y Bernard, Comrie eds. Approaches to the Typology of Word Classes. Berlin: Mouton de Gruyter, 2000.

Data recebimento: 16/03/2014.

Data aceite: 18/04/2014. 